\title{
Plantas Daninhas em Pastagens de Várzeas no Estado de MinAS Gerais ${ }^{1}$
}

\author{
Meadow Holm Pasture Weeds in Fields in the State of Minas Gerais \\ LARA, J.F.R. ${ }^{2}$, MACEDO, J.F. ${ }^{2}$ e BRANDÃO, M. ${ }^{2}$
}

\begin{abstract}
RESUMO - O controle das plantas daninhas pode onerar os custos da produção agropecuária e causar danos ao meio ambiente. Com o objetivo de conhecer a flora infestante que ocorre em pastagens formadas nas várzeas que margeiam o Rio São Francisco, foi realizado um estudo, no período de maio de 1997 a abril de 1999, nos municípios de Iguatama, Bom Despacho, Luz, Martinho Campos, Pompéu e Abaeté, localizados no Estado de Minas Gerais, Brasil. Cem amostras de $0,50 \times 0,50 \mathrm{~cm}$ foram coletadas em cada local, a partir das quais foram calculados os parâmetros freqüência, densidade, abundância, indice de valor de importância de cada espécie e índice de similaridade entre as áreas. Ao todo foram identificadas 110 espécies de plantas daninhas invasoras, pertencentes a 29 familias, sendo Asteraceae a que apresentou maior número de espécies em todos os locais. As espécies mais importantes foram: Vernonia polyanthes, em Iguatama (IVI = 69,32); Corchorus hirtus, em Bom Despacho (IVI = 54,87); Echinochloa cruz-galli, em Pompéu (IVI = 42,27); Ipomoea grandifolia, em Martinho Campos (IVI = 39,13); Hypenia densiflora, em Abaeté (IVI = 33,46); e Sida rhombifolia, em Luz (IVI = $28,41)$. O indice de similaridade calculado entre as áreas foi baixo.
\end{abstract}

Palavras-chave: forrageiras, pecuária, sustentabilidade.

ABSTRACT - Weed control can raise production costs in agriculture, causing damages to the environment. This study was carried out to investigate the weed populations occurring in pasture of six counties located along the São Francisco River, Minas Gerais, Brazil, from May 1997 to April 1999, in Iguatama, Bom Despacho, Luz, Martinho Campos, Pompéu and Abaeté. One hundred samples of $0.50 \mathrm{~m} \times 0.50 \mathrm{~m}$ from each site were used to calculate frequency, density, abundance, value of importance of each species and the similarity index among the sites. A total of 110 weed species were identified, from 29 botanical families, with the Asteraceae being the family with the greatest number of species in all the sites. The most important species in each site were Vernonia polyanthes, in Iguatama (IVI = 69.32); Corchorus hirtus, in Bom Despacho (IVI = 54.87); Echinochloa cruz-galli, in Pompéu (IVI = 42.27); Ipomoea grandifolia, in Martinho Campos (IVI = 39.13); Hypenia densiflora, in Abaeté (IVI = 33.46); and Sida rhombifolia, in Luz (IVI = 28.41). The similarity Index calculated for the localities was low.

Key words: forage, cattle productions, sustainability.

\section{INTRODUÇÃO}

Nesse momento em que governo, sociedade e ciência discutem e buscam soluções para a questão da quantidade e qualidade das águas, cresce também a responsabilidade pelo manejo adequado das áreas marginais aos cursos d'água, de forma a se evitar a contaminação do solo e dos mananciais.

Na Bacia do Rio São Francisco, em Minas Gerais, a atividade agropecuária desperta

1 Recebido para publicação em 24.5.2002 e na forma revisada em 2.4.2003.

Auxílio financeiro da Fundação de Amparo à Pesquisa do Estado de Minas Gerais - FAPEMIG

2 Pesquisadores da EPAMIG, Av. José Cândido da Silveira 1647, União, 30170-000 Belo Horizonte-MG. 
preocupação quanto aos impactos ambientais, pela sua abrangência em termos de área ocupada e por sua importância econômica na região.

Intensamente utilizadas para culturas e pastagens, as várzeas do Rio São Francisco permanecem em sua maior parte alagadas durante um período do ano, comumente nos meses de dezembro a março, mas, no período em que estão secas, tornam-se altamente produtivas, especialmente para o desenvolvimento de gramineas forrageiras. Quando se plantam espécies tolerantes ao alagamento o aproveitamento é ainda maior, pois, nas áreas baixas e úmidas, as forrageiras permanecem verdes por quase todo o inverno. Esse é o caso da gramínea forrageira Brachiaria mutica, conhecida como capim-tangola, que se adapta bem às condições de umidade das várzeas, é resistente ao alagamento e bastante aceita por bovinos e eqüinos, sendo, por isso, muito utilizada na formação de pastagens nessas áreas.

Devido às boas condições de umidade e qualidade do solo, o crescimento de plantas invasoras é favorecido, tornando-se um sério problema para os pecuaristas, na manutenção das pastagens plantadas.

Os trabalhos de Mascarenhas et al. (1992, 1995) e Cunha \& Brandão (1997) são os únicos até então encontrados que tratam das plantas invasoras em áreas de várzeas no Estado de Minas Gerais. O primeiro limita-se a espécies pertencentes às famílias Poaceae, LeguminosaePapilionoideae, Asteraceae e Cyperaceae; o segundo relata espécies pouco mencionadas para áreas de várzeas; e o terceiro trata das culturas de arroz e milho no município de Governador Valadares, Bacia do Rio Doce. Na Bacia do Rio São Francisco esse tipo de estudo é escasso, se revestindo de valor por se tratar da principal bacia hidrográfica de Minas Gerais.

O objetivo deste trabalho foi apresentar uma contribuição ao estudo da composição florística e da estrutura das populações de plantas invasoras ocorrentes em pastagens cultivadas nas várzeas do Rio São Francisco, no Estado de Minas Gerais, de forma a subsidiar pesquisadores, técnicos e pecuaristas no emprego de controles eficientes, que visem não apenas reduzir os custos, mas também promover a sustentabilidade dos sistemas agropecuários.

\section{MATERIAL E MÉTODOS}

Os levantamentos das plantas invasoras foram realizados em pastagens formadas com Brachiaria mutica nas margens do Rio São Francisco, Minas Gerais, nos municípios de Pompéu, em 2.5.1997; Abaeté, em 1.9.1997; Bom Despacho, em 27.7.1998; Luz, em 15.9.1998; Iguatama, em 25.11.1998; e Martinho Campos, em 9.4.1999. Em cada local de coleta foi arremessado, a cada 10 metros e por 100 vezes, um quadrado de madeira de 50 x $50 \mathrm{~cm}$, perfazendo um total de 100 parcelas e uma área amostral de $25 \mathrm{~m}^{2}$. A cada arremesso, foram identificadas dentro do quadrado as espécies de plantas invasoras e o número de indivíduos de cada uma delas.

Material botânico das plantas invasoras foi coletado, para identificação e confecção de exsicatas e posterior incorporação ao herbário da Empresa de Pesquisa Agropecuária de Minas Gerais - PAMG/EPAMIG.

Para análise da estrutura das comunidades de plantas invasoras foram calculados os seguintes parâmetros: freqüência das espécies informa sobre a distribuição das espécies pelas áreas; densidade - dá idéia da quantidade de plantas por unidade de área em cada espécie; abundância - informa sobre as espécies cujas plantas ocorrem concentradas em determinados pontos; freqüência relativa, densidade relativa e abundância relativa - fornecem informações de cada espécie, em relação a todas as outras encontradas nas áreas; e índice de valor de importância - indica quais espécies são mais importantes dentro da área estudada.

No cálculo desses parâmetros foram utilizadas as seguintes fórmulas (Brandão et al., 1998):

$$
\begin{aligned}
& \text { Freqüência }(\text { Fre })=\frac{\mathrm{N}^{\mathrm{o}} \text { de parcelas que contêm a espécie }}{\mathrm{N}^{\mathrm{o}} \text { total de parcelas utilizadas }} \\
& \text { Densidade }(\text { Den })=\frac{\mathrm{N}^{\mathrm{o}} \text { total de indivíduos por espécie }}{\text { Área total coletada }} \\
& \text { Abundância }(\mathrm{Abu})=\frac{\mathrm{N}^{\mathrm{o}} \text { total de indivíduos por espécie }}{\mathrm{N}^{\mathrm{o}} \text { total de parcelas que contêm a espécie }}
\end{aligned}
$$


Freqüência Relativa $($ Frr $)=\begin{gathered}\text { Freqüência da espécie } \times 100 \\ \text { Freqüência total de todas as espécies }\end{gathered}$

Densidade Relativa $($ Der $)=\begin{gathered}\text { Densidade da espécie } \times 100 \\ \text { Densidade total de todas as espécies }\end{gathered}$

Abundância Relativa $($ Abr $)=\quad$ Abundância da espécie $\times 100$ Abundância total de todas as espécies

Índice de Valor de Importância (IVI) $=$ Fre $R+$ Den R + Abu R

$\mathrm{Na}$ análise de similaridade florística entre as populações de plantas das áreas estudadas foi utilizado o Îndice de Similaridade de Sథrensen (Sథrensen, 1972). Para o seu cálculo utilizou-se a seguinte fórmula:

Índice de Similaridade (IS) $=2 \mathrm{a} / \mathrm{b}+\mathrm{c}$

em que: $\mathrm{a}=$ número de espécies comuns às duas áreas; e b, c = número total de espécies nas duas áreas comparadas.

O "IS" varia de 0 a 1 , sendo máximo quando todas as espécies são comuns às duas áreas $\mathrm{e}$ mínimo quando não há espécies comuns.

\section{RESULTADOS E DISCUSSÃO}

A composição florística e os parâmetros fitossociológicos calculados para cada localidade são apresentados nas Tabelas 1 a 8 .

Tabela 1 - Número de espécies de plantas invasoras em cada família, coletadas em pastagens de várzeas nas margens do Rio São Francisco, em Minas Gerais

\begin{tabular}{|c|c|c|c|c|c|c|c|}
\hline \multirow[b]{2}{*}{ Família } & \multicolumn{6}{|c|}{ Número de espécies de plantas invasoras por família e por localidade } & \multirow[b]{2}{*}{ Tota } \\
\hline & Iguatama & $\begin{array}{c}\text { Bom } \\
\text { Despacho }\end{array}$ & $\begin{array}{l}\text { Martinho } \\
\text { Campos }\end{array}$ & Luz & Abaeté & Pompéu & \\
\hline Asteraceae & 08 & 11 & 10 & 07 & 10 & 09 & 55 \\
\hline Malvaceae & 04 & 01 & 05 & 07 & 08 & 03 & 28 \\
\hline Poaceae & 03 & 03 & 03 & 02 & 01 & 04 & 16 \\
\hline Euphorbiaceae & 03 & 04 & 01 & 03 & 01 & 01 & 13 \\
\hline Tiliaceae & 02 & 01 & 01 & 02 & 02 & 01 & 09 \\
\hline Leguminosae - Caes. & 02 & 01 & & 01 & 01 & 01 & 06 \\
\hline Leguminosae - Pap. & 02 & 01 & 03 & 02 & 05 & 02 & 15 \\
\hline Lamiaceae & 01 & & 03 & 04 & 04 & 02 & 14 \\
\hline Convolvulaceae & 01 & 01 & 02 & 03 & 02 & 02 & 11 \\
\hline Myrtaceae & 01 & & 01 & & & & 02 \\
\hline Verbenaceae & 01 & 01 & 01 & 01 & 01 & 01 & 06 \\
\hline Sterculiaceae & 01 & 01 & 01 & 01 & 01 & 01 & 06 \\
\hline Amaranthaceae & 01 & & & 01 & 02 & & 04 \\
\hline Capparaceae & 01 & & & & & 01 & 02 \\
\hline Oxalidaceae & 01 & & & & & & 01 \\
\hline Maranthaceae & 01 & & & & & & 01 \\
\hline Solanaceae & & 04 & 02 & 01 & 03 & 03 & 13 \\
\hline Boraginaceae & & 01 & & & 01 & & 02 \\
\hline Lythraceae & & & 01 & & 01 & & 02 \\
\hline Cyperaceae & & & 01 & & & & 01 \\
\hline Scrophulariaceae & & & 01 & & 01 & & 02 \\
\hline Rubiaceae & & & 01 & 02 & 02 & 01 & 06 \\
\hline Apiaceae & & & & 01 & & & 01 \\
\hline Asclepiadaceae & & & & 01 & 01 & & 02 \\
\hline Cannaceae & & & & 01 & & & 01 \\
\hline Sapindaceae & & & & 01 & & & 01 \\
\hline Commelinaceae & & & & 01 & 01 & & 02 \\
\hline Brassicaceae & & & & 01 & & & 01 \\
\hline Cucurbitaceae & & & & & & 02 & 02 \\
\hline Leguminosae-Mim. & & & & & & 01 & 01 \\
\hline Budlejaceae & & & & & & 01 & 01 \\
\hline Total & 33 & 30 & 37 & 43 & 48 & 36 & 227 \\
\hline
\end{tabular}


Tabela 2 - Espécies de plantas invasoras encontradas em pastagens formadas nas várzeas do Rio São Francisco, município de Iguatama, Minas Gerais

\begin{tabular}{|c|c|c|c|c|c|c|c|c|c|c|}
\hline Espécie & Família & $\begin{array}{l}\mathrm{N}^{\mathrm{o}} \text { de } \\
\text { Parc. }\end{array}$ & $\begin{array}{l}\mathrm{N}^{\mathrm{o}} \text { de } \\
\text { Indiv. }\end{array}$ & Fre & Den & Abu & Frr & Der & Abr & IVI \\
\hline Vernonia polyanthes Less. & Asteraceae & 53 & 161 & 0,53 & 6,44 & 3,04 & 25,36 & 38,61 & 5,55 & 69,52 \\
\hline Corchorus hirtus L. & Tiliaceae & 18 & 53 & 0,18 & 2,12 & 2,94 & 8,61 & 12,71 & 5,38 & 26,70 \\
\hline Chamaesyce hirta (L.) Millsp. & Euphorbiaceae & 21 & 35 & 0,21 & 1,40 & 1,67 & 10,05 & 8,39 & 3,04 & 21,49 \\
\hline Conyza bonariensis (L.) Cronquist & Asteraceae & 18 & 31 & 0,18 & 1,24 & 1,72 & 8,61 & 7,43 & 3,15 & 19,19 \\
\hline Sida glaziovii K.Schum. & Malvaceae & 15 & 17 & 0,15 & 0,68 & 1,13 & 7,18 & 4,08 & 2,07 & 13,32 \\
\hline Emilia sonchifolia (L.) DC. & Asteraceae & 15 & 15 & 0,15 & 0,60 & 1,00 & 7,18 & 3,60 & 1,83 & 12,60 \\
\hline Phyllanthus tenellus Roxb. & Euphorbiaceae & 2 & 10 & 0,02 & 0,40 & 5,00 & 0,96 & 2,40 & 9,13 & 12,49 \\
\hline Sida rhombifolia L. & Malvaceae & 9 & 14 & 0,09 & 0,56 & 1,56 & 4,31 & 3,36 & 2,84 & 10,51 \\
\hline Senna obtusifolia (L.) H. S. Irwin \& Bareby & Leguminosae-Caes. & 9 & 13 & 0,09 & 0,52 & 1,44 & 4,31 & 3,12 & 2,64 & 10,06 \\
\hline Sida urens L. & Malvaceae & 3 & 9 & 0,03 & 0,36 & 3,00 & 1,44 & 2,16 & 5,48 & 9,07 \\
\hline Oxalis corniculata L. & Oxalidaceae & 1 & 4 & 0,01 & 0,16 & 4,00 & 0,48 & 0,96 & 7,31 & 8,74 \\
\hline Ageratum conyzoides $\mathrm{L}$. & Asteraceae & 8 & 8 & 0,08 & 0,32 & 1,00 & 3,83 & 1,92 & 1,83 & 7,57 \\
\hline Paspalum notatum Flügge' & Poaceae & 6 & 8 & 0,06 & 0,32 & 1,33 & 2,87 & 1,92 & 2,44 & 7,23 \\
\hline Acanthospermum australe (Loefl.) Kuntze & Asteraceae & 5 & 7 & 0,05 & 0,28 & 1,40 & 2,39 & 1,68 & 2,56 & 6,63 \\
\hline Chamaesyce hyssopifolia (L.) Small & Euphorbiacea & 1 & 3 & 0,01 & 0,12 & 3,00 & 0,48 & 0,72 & 5,48 & 6,68 \\
\hline Ipomoea grandifolia (Dammer)O'Donel & Convolvulaceae & 4 & 4 & 0,04 & 0,16 & 1,00 & 1,91 & 0,96 & $\begin{array}{l}1,83 \\
1,4\end{array}$ & 4,70 \\
\hline Waltheria americana $\mathrm{L}$. & Sterculiaceae & 1 & 2 & 0,01 & 0,08 & 2,00 & 0,48 & 0,48 & 3,65 & 4,61 \\
\hline Eupatorium pauciflorum. Kunth. & Asteraceae & 1 & 2 & 0,01 & 0,08 & 2,00 & 0,48 & 0,48 & $\begin{array}{l}3,65 \\
3,65\end{array}$ & 4,61 \\
\hline Elephantopus mollis Kunth & Asteraceae & 1 & 2 & 0,01 & 0,08 & 2,00 & 0,48 & 0,48 & 3,65 & 4,61 \\
\hline Hyparrhenia rufa (Ness.) Stapf & Poaceae & 2 & 3 & 0,02 & 0,12 & 1,50 & 0,96 & 0,72 & 2,74 & 4,42 \\
\hline Amaranthus lividus L. & Amaranthaceae & 3 & 3 & 0,03 & 0,12 & 1,00 & 1,44 & 0,72 & 1,83 & 3,98 \\
\hline Andropogon bicornis L. & Poaceae & 2 & 2 & 0,02 & 0,08 & 1,00 & 0,96 & 0,48 & 1,83 & 3,26 \\
\hline Stachytarpheta cayennensis (Rich.) M. Vahl & Verbenaceae & 1 & 1 & 0,01 & 0,04 & 1,00 & 0,48 & 0,24 & 1,83 & 2,55 \\
\hline Stylosanthes guianensis (Aubl.)Sw. & Leguminosae-Pap. & 1 & 1 & 0,01 & 0,04 & 1,00 & 0,48 & 0,24 & 1,83 & 2,55 \\
\hline Sida carpinifolia L.f. & Malvaceae & 1 & 1 & 0,01 & 0,04 & 1,00 & 0,48 & 0,24 & 1,83 & 2,55 \\
\hline Senna occidentalis (L.) Link. & Leguminosae-Caes. & 1 & 1 & 0,01 & 0,04 & 1,00 & 0,48 & 0,24 & 1,83 & \begin{tabular}{l}
2,55 \\
2,55 \\
\hdashline
\end{tabular} \\
\hline Pterocaulon lanatum Kuntze & Asteraceae & 1 & 1 & 0,01 & 0,04 & 1,00 & 0,48 & 0,24 & 1,83 & 2,55 \\
\hline Psidium guajava L. & Myrtaceae & 1 & 1 & 0,01 & 0,04 & 1,00 & 0,48 & 0,24 & 1,83 & 2,55 \\
\hline Hyptis suaveolens (L.) Poit. & Lamiaceae & 1 & 1 & 0,01 & 0,04 & 1,00 & 0,48 & 0,24 & 1,83 & 2,55 \\
\hline Desmodium incanum DC. & Leguminosae-Pap. & 1 & 1 & 0,01 & 0,04 & 1,00 & 0,48 & 0,24 & 1,83 & 2,55 \\
\hline Cleome spinosa Jacq. & Capparaceae & 1 & 1 & 0,01 & 0,04 & 1,00 & 0,48 & 0,24 & 1,83 & 2,55 \\
\hline Thalia geniculata L. & Maranthaceae & 1 & 1 & 0,01 & 0,04 & 1,00 & 0,48 & 0,24 & 1,83 & 2,55 \\
\hline Triumfetta semitriloba Jacq. & Tiliaceae & 1 & 1 & 0,01 & 0,04 & 1,00 & 0,48 & 0,24 & 1,83 & 2,55 \\
\hline $\begin{array}{r}\text { Total } \\
\end{array}$ & & & & 2,09 & 16,68 & 54,74 & 100,00 & 100,00 & 100,00 & 300,00 \\
\hline
\end{tabular}

№ de Par. = número de parcelas; № de Indiv. = número de indivíduos; Fre = freqüência; Den $=$ densidade; Abu $=$ abundância; Frr $=$ freqüência relativa; Der $=$ densidade relativa; $\mathrm{Abr}=$ abundância relativa; IVI = índice de valor de importância.

Foram identificadas 110 espécies de plantas invasoras, pertencentes a 29 famílias botânicas (Tabela 1). A família Asteraceae apresentou o maior número de espécies nos seis locais. Nesta família estão incluídas três espécies que ocorreram em todos os locais: Ageratum conyzoides, Emilia sonchifolia e Vernonia polyanthes. Mais da metade das famílias em cada município estão representadas por apenas uma espécie (Tabela 1). Asteraceae aparece também com maior número de espécies no trabalho de Cunha \& Brandão (1997).
Vernonia polyanthes foi a espécie mais importante no municipio de Iguatama, com IVI igual a 69,52 (Tabela 2). A espécie Corchorus hirtus (Tiliaceae) sobressaiu em Bom Despacho, com IVI igual a 54,87 (Tabela 3). Em Martinho Campos, o destaque foi para a espécie Ipomoea grandifolia (Convolvulaceae), cujo IVI atingiu 38,13 (Tabela 4). A família Malvaceae aparece em destaque no município de Luz, com Sida rhombifolia, que apresentou IVI igual a 28,41 (Tabela 5). Em Pompéu, a espécie Echinchloa cruz-galli (Poaceae) foi a mais importante, com IVI igual a 42,27 (Tabela 6), e, em Abaeté, 
Hypenia densiflora (Lamiaceae), com IVI igual a 33,46 (Tabela 7), foi a espécie mais importante.

A família Malvaceae, além de incluir a espécie mais importante no municipio de Luz, aparece em segundo lugar com maior número de espécies em Iguatama, Martinho Campos, Luz e Abaeté.

É importante observar o parâmetro abundância (Abu), que revela as espécies que apareceram em reboleira, como Phyllanthus tenellus e Oxalis corniculata, em Iguatama, Conyza bonariensis, em Luz, Sida linifolia, em Pompéu, e Desmodium incanum, em Abaeté, que podem admitir métodos próprios para seu controle.

A similaridade é baixa entre as localidades estudadas (Tabela 8). Muitas espécies foram exclusivas em cada local. Espécies que hoje são raras poderão ser importantes no futuro, pois observou-se que, das espécies citadas por Mascarenhas et al. (1995) como raras em áreas de várzeas, algumas constam deste trabalho e se destacam em importância, como é o caso de Ipomoea grandifolia (=I. aristolochiaefolia H.B.K.), em Martinho Campos e em Bom Despacho; Corchorus hirtus, em Iguatama; Leonurus sibiricus, em Luz; e Sida urens, em Abaeté.

Tabela 3 - Espécies de plantas daninhas encontradas em pastagens formadas nas várzeas do Rio São Francisco, município de Bom Despacho, Minas Gerais

\begin{tabular}{|c|c|c|c|c|c|c|c|c|c|c|}
\hline Espécie & Família & $\begin{array}{l}\mathrm{N}^{\underline{0}} \text { de } \\
\text { Parc. }\end{array}$ & $\begin{array}{l}\mathrm{N}^{\mathrm{o}} \mathrm{de} \\
\text { Indiv. }\end{array}$ & Fre & Den & Abu & Frr & Der & Abr & IVI \\
\hline Corchorus hirtus L. & Tiliaceae & 32 & 94 & 0,32 & 3,76 & 2,94 & 18,08 & 30,62 & 6,17 & 54,87 \\
\hline Ipomoea grandifolia (Dammer) O`Donel & Convolvulaceae & 31 & 43 & 0,31 & 1,72 & 1,39 & 17,51 & 14,01 & 2,91 & 34,43 \\
\hline Conyza bonariensis (L.) Cronquist & Asteraceae & 11 & 25 & 0,11 & 1,00 & 2,27 & 6,21 & 8,14 & 4,77 & 19,13 \\
\hline Phyllanthus tenellus Roxb. & Euphorbiaceae & 12 & 12 & 0,12 & 0,48 & 1,00 & 6,78 & 3,91 & 2,10 & 12,79 \\
\hline Acanthospermum australe (Loefl.) Kunt. & Asteraceae & 11 & 12 & 0,11 & 0,48 & 1,09 & 6,21 & 3,91 & 2,29 & 12,41 \\
\hline Spilanthes acmella (L.) Murray & Asteraceae & 10 & 12 & 0,1 & 0,48 & 1,20 & 5,65 & 3,91 & 2,52 & 12,08 \\
\hline Emilia sonchifolia (L.) DC & Asteraceae & 6 & 14 & 0,06 & 0,56 & 2,33 & 3,39 & 4,56 & 4,90 & 12,85 \\
\hline Bidens pilosa $\mathrm{L}$. & Asteraceae & 7 & 11 & 0,07 & 0,44 & 1,57 & 3,95 & 3,58 & 3,30 & 10,84 \\
\hline Paspalum notatum Flüggé & Poaceae & 8 & 8 & 0,08 & 0,32 & 1,00 & 4,52 & 2,61 & 2,10 & 9,23 \\
\hline Ageratum conyzoides $\mathrm{L}$. & Asteraceae & 3 & 12 & 0,03 & 0,48 & 4,00 & 1,69 & 3,91 & 8,40 & 14,00 \\
\hline Heliotropium indicum L. & Boraginaceae & 4 & 9 & 0,04 & 0,36 & 2,25 & 2,26 & 2,93 & 4,72 & 9,92 \\
\hline Eleusine indica (L.) Gaertn. & Poaceae & 4 & 9 & 0,04 & 0,36 & 2,25 & 2,26 & 2,93 & 4,72 & 9,92 \\
\hline Waltheria americana $\mathrm{L}$. & Sterculiaceae & 6 & 6 & 0,06 & 0,24 & 1,00 & 3,39 & 1,95 & 2,10 & 7,44 \\
\hline Eclipta alba (L.) Hassk. & Asteraceae & 5 & 5 & 0,05 & 0,2 & 1,00 & 2,82 & 1,63 & 2,10 & 6,55 \\
\hline Solanum palinacanthum Dunal & Solanaceae & 3 & 6 & 0,03 & 0,24 & 2,00 & 1,69 & 1,95 & 4,20 & 7,85 \\
\hline Lantana trifolia $\mathrm{L}$. & Verbenaceae & 3 & 4 & 0,03 & 0,16 & 1,33 & 1,69 & 1,30 & 2,80 & 5,80 \\
\hline Andropogon bicornis L. & Poaceae & 3 & 3 & 0,03 & 0,12 & 1,00 & 1,69 & 0,98 & 2,10 & 4,77 \\
\hline Chamaesyce hyssopifolia (L.) Small & Euphorbiaceae & 1 & 3 & 0,01 & 0,12 & 3,00 & 0,56 & 0,98 & 6,30 & 7,84 \\
\hline Vernonia remotiflora Rich. & Asteraceae & 2 & 2 & 0,02 & 0,08 & 1,00 & 1,13 & 0,65 & 2,10 & 3,88 \\
\hline Sp indeterminada & & 2 & 2 & 0,02 & 0,08 & 1,00 & 1,13 & 0,65 & 2,10 & 3,88 \\
\hline Solanum americanum Mill. & Solanaceae & 2 & 2 & 0,02 & 0,08 & 1,00 & 1,13 & 0,65 & 2,10 & 3,88 \\
\hline Solanum aculeatissimum Jacq. & Solanaceae & 2 & 2 & 0,02 & 0,08 & 1,00 & 1,13 & 0,65 & 2,10 & 3,88 \\
\hline Eupatorium pauciflorum Kunth & Asteraceae & 1 & 2 & 0,01 & 0,08 & 2,00 & 0,56 & 0,65 & 4,20 & 5,42 \\
\hline Desmodium incanum DC. & Leguminosae-Pap. & 1 & 2 & 0,01 & 0,08 & 2,00 & 0,56 & 0,65 & 4,20 & 5,42 \\
\hline Sida carpinifolia L.f. & Malvaceae & 1 & 1 & 0,01 & 0,04 & 1,00 & 0,56 & 0,33 & 2,10 & 2,90 \\
\hline Senna occidentalis (L.) Link & Leguminosae-Caes. & 1 & 1 & 0,01 & 0,04 & 1,00 & 0,56 & 0,33 & 2,10 & 2,99 \\
\hline Ricinus communis $\mathrm{L}$. & Euphorbiaceae & 1 & 1 & 0,01 & 0,04 & 1,00 & 0,56 & 0,33 & 2,10 & 2,90 \\
\hline Nicandra physalodes (L.) Gaertn. & Solanaceae & 1 & 1 & 0,01 & 0,04 & 1,00 & 0,56 & 0,33 & 2,10 & 2,99 \\
\hline Euphorbia heterophylla L. & Euhorbiaceae & 1 & 1 & 0,01 & 0,04 & 1,00 & 0,56 & 0,33 & 2,10 & 2,90 \\
\hline Blainvillea biaristata DC. & Asteraceae & 1 & 1 & 0,01 & 0,04 & 1,00 & 0,56 & 0,33 & 2,10 & 2,90 \\
\hline Acanthospermum hispidum DC. & Asteraceae & 1 & 1 & 0,01 & 0,04 & 1,00 & 0,56 & 0,33 & 2,10 & 2,99 \\
\hline \begin{tabular}{|c|} 
TOTAL \\
\end{tabular} & & & & 1,77 & 12,28 & 47,63 & 100,00 & 100,00 & 100,00 & 300,00 \\
\hline
\end{tabular}

№ de Par. = número de parcelas; № de Indiv. = número de indivíduos; Fre = freqüência; Den = densidade; Abu = abundância; Frr = frequiência relativa; Der $=$ densidade relativa; $\mathrm{Abr}=$ abundância relativa; IVI = índice de valor de importância. 
Tabela 4 - Espécies de plantas daninhas encontradas em pastagens formadas nas várzeas do Rio São Francisco, município de Martinho Campos, Minas Gerais

\begin{tabular}{|c|c|c|c|c|c|c|c|c|c|c|}
\hline Espécie & Família & $\begin{array}{l}\mathrm{N}^{\mathrm{o}} \mathrm{de} \\
\text { Parc. }\end{array}$ & $\begin{array}{l}\mathrm{N}^{\mathrm{o}} \text { de } \\
\text { Indiv. }\end{array}$ & Fre & Den & Abu & Frr & Der & Abr & IVI \\
\hline Ipomoea grandifolia (Dammer) O`Donel & Convolvulaceae & 49 & 77 & 0,49 & 3,08 & 1,57 & 17,44 & 17,70 & 2,99 & 38,13 \\
\hline Conyza bonariensis (L.) Cronquist & Asteraceae & 29 & 62 & 0,29 & 2,48 & 2,14 & 10,32 & 14,25 & 4,07 & 28,64 \\
\hline Sida glaziovii K. Schum. & Malvaceae & 18 & 38 & 0,18 & 1,52 & 2,11 & 6,41 & 8,74 & 4,02 & 19,16 \\
\hline Phyllanthus tenellus Roxb. & Euphorbiaceae & 22 & 25 & 0,22 & 1,00 & 1,14 & 7,83 & 5,75 & 2,16 & 15,74 \\
\hline Ageratum conyzoides $\mathrm{L}$. & Asteraceae & 15 & 27 & 0,15 & 1,08 & 1,80 & 5,34 & 6,21 & 3,43 & 14,97 \\
\hline Vernonia polyanthes Lees. & Asteraceae & 10 & 21 & 0,10 & 0,84 & 2,10 & 3,56 & 4,83 & 4,00 & 12,38 \\
\hline Ipomoea hederifolia $\mathrm{L}$. & Convolvulaceae & 15 & 19 & 0,15 & 0,76 & 1,27 & 5,34 & 4,37 & 2,41 & 12,12 \\
\hline Cyperus rotundus $\mathrm{L}$. & Cyperaceae & 6 & 18 & 0,06 & 0,72 & 3,00 & 2,14 & 4,14 & 5,71 & 11,98 \\
\hline Leonotis nepetaefolia (R. Br.) W. T. Aiton & Lamiaceae & 12 & 19 & 0,12 & 0,76 & 1,58 & 4,27 & 4,37 & 3,01 & 11,65 \\
\hline Emilia sonchifolia $($ L.) DC. & Asteraceae & 12 & 16 & 0,12 & 0,64 & 1,33 & 4,27 & 3,68 & 2,54 & 10,49 \\
\hline Sida urens $\mathrm{L}$. & Malvaceae & 8 & 13 & 0,08 & 0,52 & 1,63 & 2,85 & 2,99 & 3,09 & 8,93 \\
\hline Stachytarpheta cayennensis (Rich.) M. Vahl. & Verbenaceae & 1 & 4 & 0,01 & 0,16 & 4,00 & 0,36 & 0,92 & 7,61 & 8,89 \\
\hline Physalis angulata $\mathrm{L}$. & Solanaceae & 11 & 12 & 0,11 & 0,48 & 1,09 & 3,91 & 2,76 & 2,08 & 8,75 \\
\hline Blainvillea rhomboidea Cass. & Asteraceae & 2 & 6 & 0,02 & 0,24 & 3,00 & 0,71 & 1,38 & 5,71 & 7,80 \\
\hline Vernonia remotiflora Rich. & Asteraceae & 8 & 9 & 0,08 & 0,36 & 1,13 & 2,85 & 2,07 & 2,14 & 7,06 \\
\hline Paspalum notatum Flüggé & Poaceae & 5 & 7 & 0,05 & 0,28 & 1,40 & 1,78 & 1,61 & 2,66 & 6,05 \\
\hline Eclipta alba (L.) Hassk. & Asteraceae & 6 & 6 & 0,06 & 0,24 & 1,00 & 2,14 & 1,38 & 1,90 & 5,42 \\
\hline Stylosanthes acuminata M.B.Ferreira et S. Costa & Leguminosae-Pap. & 6 & 6 & 0,06 & 0,24 & 1,00 & 2,14 & 1,38 & 1,90 & 5,42 \\
\hline Zornia reticulata $\mathrm{Sm}$. & Leguminosae-Pap. & 6 & 6 & 0,06 & 0,24 & 1,00 & 2,14 & 1,38 & 1,90 & 5,42 \\
\hline Nicandra physalodes (L.) Gaertn. & Solanaceae & 4 & 5 & 0,04 & 0,20 & 1,25 & 1,42 & 1,15 & 2,38 & 4,95 \\
\hline Sida cordifolia $\mathrm{L}$. & Malvaceae & 4 & 5 & 0,04 & 0,20 & 1,25 & 1,42 & 1,15 & 2,38 & 4,95 \\
\hline Waltheria americana $\mathrm{L}$. & Sterculiaceae & 4 & 5 & 0,04 & 0,20 & 1,25 & 1,42 & 1,15 & 2,38 & 4,95 \\
\hline Eleusine indica (L.) Gaertn. & Poaceae & 5 & 5 & 0,05 & 0,20 & 1,00 & 1,78 & 1,15 & 1,90 & 4,83 \\
\hline Galinsoga parviflora Cav. & Asteraceae & 2 & 3 & 0,02 & 0,12 & 1,50 & 0,71 & 0,69 & 2,86 & 4,26 \\
\hline Marsypianthes chamaedrys (Vahl) Kuntze & Lamiaceae & 4 & 4 & 0,04 & 0,16 & 1,00 & 1,42 & 0,92 & 1,90 & 4,25 \\
\hline Desmodium barbatum (L.) Benth. & Leguminosae-Pap. & 3 & 3 & 0,03 & 0,12 & 1,00 & 1,07 & 0,69 & 1,90 & 3,66 \\
\hline Panicum maximum Jacq. & Poaceae & 3 & 3 & 0,03 & 0,12 & 1,00 & 1,07 & 0,69 & 1,90 & 3,66 \\
\hline Scoparia dulcis $\mathrm{L}$. & Scrophulariaceae & 2 & 2 & 0,02 & 0,08 & 1,00 & 0,71 & 0,46 & 1,90 & 3,08 \\
\hline Cuphea carthagenensis (Jacq.) J.F. Macbr. & Lythraceae & 1 & 1 & 0,01 & 0,04 & 1,00 & 0,36 & 0,23 & 1,90 & 2,49 \\
\hline Eupatorium pauciflorum Kunth & Asteraceae & 1 & 1 & 0,01 & 0,04 & 1,00 & 0,36 & 0,23 & 1,90 & 2,49 \\
\hline Hyptis suaveolens (L.) Poit. & Lamiaceae & 1 & 1 & 0,01 & 0,04 & 1,00 & 0,36 & 0,23 & 1,90 & 2,49 \\
\hline Porophyllum ruderale (Jacq.) Cass. & Asteraceae & 1 & 1 & 0,01 & 0,04 & 1,00 & 0,36 & 0,23 & 1,90 & 2,49 \\
\hline Psidium guajava $\mathrm{L}$. & Myrtaceae & 1 & 1 & 0,01 & 0,04 & 1,00 & 0,36 & 0,23 & 1,90 & 2,49 \\
\hline Sida carpinifolia L.f. & Malvaceae & 1 & 1 & 0,01 & 0,04 & 1,00 & 0,36 & 0,23 & 1,90 & 2,49 \\
\hline Sida cerradoensis Krapov. & Malvaceae & 1 & 1 & 0,01 & 0,04 & 1,00 & 0,36 & 0,23 & 1,90 & 2,49 \\
\hline Spermacoce verticillata $\mathrm{L}$. & Rubiaceae & 1 & 1 & 0,01 & 0,04 & 1,00 & 0,36 & 0,23 & 1,90 & 2,49 \\
\hline Triumfetta semitriloba Jacq. & Tiliaceae & 1 & 1 & 0,01 & 0,04 & 1,00 & 0,36 & 0,23 & 1,90 & 2,49 \\
\hline Total & & & & 2,81 & 17,40 & 52,53 & 100,00 & 100,00 & 100,00 & 300,00 \\
\hline
\end{tabular}

№ de Par. = número de parcelas; № de Indiv. = número de indivíduos; Fre = freqüência; Den = densidade; Abu = abundância; Frr = frequiência relativa; Der $=$ densidade relativa; $\mathrm{Abr}=$ abundância relativa; IVI = índice de valor de importância. 
Tabela 5 - Espécies de plantas daninhas encontradas em pastagens formadas nas várzeas do Rio São Francisco, município de Luz, Minas Gerais

\begin{tabular}{|c|c|c|c|c|c|c|c|c|c|c|}
\hline Espécie & Família & $\begin{array}{l}\mathrm{N}^{\mathrm{o}} \mathrm{de} \\
\text { Parc. }\end{array}$ & $\begin{array}{l}\text { № de } \\
\text { Indiv. }\end{array}$ & Fre & Den & Abu & Frr & Der & Abr & IVI \\
\hline Sida rhombifolia $\mathrm{L}$. & Malvaceae & 32 & 79 & 0,32 & 3,16 & 2,47 & 10,03 & 14,91 & 3,47 & 28,41 \\
\hline Leonurus sibiricus $\mathrm{L}$. & Lamiaceae & 24 & 72 & 0,24 & 2,88 & 3,00 & 7,52 & 13,58 & 4,21 & 25,32 \\
\hline Sida viarum A. St. Hill. & Malvaceae & 37 & 41 & 0,37 & 1,64 & 1,11 & 11,60 & 7,74 & 1,56 & 20,89 \\
\hline Chamaesyce hirta (L.) Mill. & Euphorbiaceae & 32 & 42 & 0,32 & 1,68 & 1,31 & 10,03 & 7,92 & $\begin{array}{l}1,84 \\
. .84\end{array}$ & $\begin{array}{l}19,80 \\
19\end{array}$ \\
\hline Conyza bonariensis (L.) Cronquist & Asteraceae & 2 & 17 & 0,02 & 0,68 & 8,50 & 0,63 & 3,21 & 11,94 & 15,78 \\
\hline Emilia sonchifolia (L.) DC. & Asteraceae & 24 & 31 & 0,24 & 1,24 & 1,29 & 7,52 & 5,85 & 1,81 & 15,19 \\
\hline 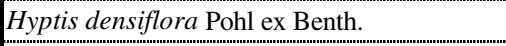 & Lamiaceae & 21 & 27 & 0,21 & 1,08 & 1,29 & 6,58 & 5,09 & 1,81 & 13,48 \\
\hline Sida urens L. & Malvaceae & 17 & 28 & 0,17 & 1,12 & 1,65 & 5,33 & 5,28 & 2,31 & 12,93 \\
\hline Ageratum conyzoides $\mathrm{L}$. & Asteraceae & 10 & 28 & 0,10 & 1,12 & 2,80 & 3,13 & 5,28 & 3,93 & 12,35 \\
\hline Ipomoea grandifolia (Dammer) O'Donel & Convolvulaceae & 10 & 28 & 0,10 & 1,12 & 2,80 & 3,13 & 5,28 & 3,93 & 12,35 \\
\hline Senna obtusifolia (L.) H.S. Irwin \& Barneby & Leguminosae-Caes. & 16 & 20 & 0,16 & 0,80 & 1,25 & 5,02 & 3,77 & 1,76 & 10,55 \\
\hline Vernonia polyanthes Lees. & Asteraceae & 7 & 19 & 0,07 & 0,76 & 2,71 & 2,19 & 3,58 & 3,81 & 9,59 \\
\hline Sida glaziovii K. Schum. & Malvaceae & 11 & 11 & 0,11 & 0,44 & 1,00 & 3,45 & 2,08 & 1,40 & 6,93 \\
\hline Leonotis nepetaefolia (R.Br.) W.T.Aiton & Lamiaceae & 1 & 4 & 0,01 & 0,16 & 4,00 & 0,31 & 0,75 & 5,62 & 6,69 \\
\hline Eclipta alba (L.) Hassk. & Asteraceae & 8 & 8 & 0,08 & 0,32 & 1,00 & 2,51 & 1,51 & 1,40 & 5,42 \\
\hline Stachytarpheta cayennensis (Rich.) M. Vahl & Verbenaceae & 1 & 3 & 0,01 & 0,12 & 3,00 & 0,31 & 0,57 & 4,21 & 5,09 \\
\hline Corchorus hirtus L. $\quad$. & Tiliaceae & 6 & 6 & 0,06 & 0,24 & 1,00 & 1,88 & 1,13 & 1,40 & 4,42 \\
\hline Ricinus communis L. & Euphorbiaceae & 5 & 5 & 0,05 & 0,20 & 1,00 & 1,57 & 0,94 & 1,40 & 3,92 \\
\hline Sonchus oleraceus L. & Asteraceae & 5 & 5 & 0,05 & 0,20 & 1,00 & 1,57 & 0,94 & 1,40 & 3,92 \\
\hline Amaranthus lividus $\mathrm{L}$. & Amaranthaceae & 3 & 4 & 0,03 & 0,16 & 1,33 & 0,94 & 0,75 & 1,87 & 3,57 \\
\hline Hyptis lophantha Mart. ex Benth. & Lamiaceae & 3 & 4 & 0,03 & 0,16 & 1,33 & 0,94 & 0,75 & 1,87 & 3,57 \\
\hline Lepidium virginicum L. & Brassicaceae & 3 & 4 & 0,03 & 0,16 & 1,33 & 0,94 & 0,75 & 1,87 & 3,57 \\
\hline Merremia cissoides (Lam.) Hallier.f. & Convolvulaceae & 1 & 2 & 0,01 & 0,08 & 2,00 & 0,31 & 0,38 & 2,81 & 3,50 \\
\hline Sida cordifolia $\mathrm{L}$. & Malvaceae & 4 & 4 & 0,04 & 0,16 & 1,00 & 1,25 & 0,75 & 1,40 & 3,41 \\
\hline Vernonia remotiflora Rich. & Asteraceae & 4 & 4 & 0,04 & 0,16 & 1,00 & 1,25 & 0,75 & 1,40 & 3,41 \\
\hline Galinsoga parviflora Cav. & Asteraceae & 2 & 3 & 0,02 & 0,12 & 1,50 & 0,63 & 0,57 & 2,11 & 3,30 \\
\hline Ipomoea hederifolia L. & Convolvulaceae & 2 & 3 & 0,02 & 0,12 & 1,50 & 0,63 & 0,57 & 2,11 & 3,30 \\
\hline Canna indica $\mathrm{L}$. & Cannaceae & 3 & 3 & 0,03 & 0,12 & 1,00 & 0,94 & 0,57 & 1,40 & 2,91 \\
\hline Commelina benghalensis L. & Commelinaceae & 3 & 3 & 0,03 & 0,12 & 1,00 & 0,94 & 0,57 & 1,40 & 2,91 \\
\hline Panicum maximum Jacq. & Poaceae & 3 & 3 & 0,03 & 0,12 & 1,00 & 0,94 & 0,57 & 1,40 & 2,91 \\
\hline Indigofera suffruticosa Mill.. & Leguminosae-Pap. & 2 & 2 & 0,02 & 0,08 & 1,00 & 0,63 & 0,38 & 1,40 & 2,41 \\
\hline Sida carpinifolia L.f. & Malvaceae & 2 & 2 & 0,02 & 0,08 & 1,00 & 0,63 & 0,38 & 1,40 & 2,41 \\
\hline Solanum aculeatissimum Jacq. & Solanaceae & 2 & 2 & 0,02 & 0,08 & 1,00 & 0,63 & 0,38 & 1,40 & 2,41 \\
\hline Waltheria americana $\mathrm{L}$. & Sterculiaceae & 2 & 2 & 0,02 & 0,08 & 1,00 & 0,63 & 0,38 & 1,40 & 2,41 \\
\hline Apium leptophyllum (Pers.)F.Muell-ex Benth & Apiaceae & 1 & 1 & 0,01 & 0,04 & 1,00 & 0,31 & 0,19 & 1,40 & 1,91 \\
\hline Asclepias curassavica L. & Asclepiadaceae & 1 & 1 & 0,01 & 0,04 & 1,00 & 0,31 & 0,19 & 1,40 & 1,91 \\
\hline Cardiospermum halicacabum L. & Sapindaceae & 1 & 1 & 0,01 & 0,04 & 1,00 & 0,31 & 0,19 & 1,40 & 1,91 \\
\hline 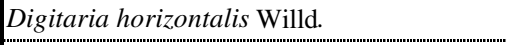 & Poaceae & 1 & 1 & 0,01 & 0,04 & 1,00 & 0,31 & 0,19 & 1,40 & 1,91 \\
\hline Chamaesyce hyssopifolia (L.) Small & Euphorbiaceae & 1 & 1 & 0,01 & 0,04 & 1,00 & 0,31 & 0,19 & 1,40 & 1,91 \\
\hline Manettia ignita (Vell.) K. Schum. & Rubiaceae & 1 & 1 & 0,01 & 0,04 & 1,00 & 0,31 & 0,19 & 1,40 & 1,91 \\
\hline Richardia scabra L. & Rubiaceae & 1 & 1 & 0,01 & 0,04 & 1,00 & 0,31 & 0,19 & 1,40 & 1,91 \\
\hline Sida santaremensis Monteiro & Malvaceae & 1 & 1 & 0,01 & 0,04 & 1,00 & 0,31 & 0,19 & 1,40 & 1,91 \\
\hline Stylosanthes scabra Vogel & Leguminosae-Pap. & 1 & 1 & 0,01 & 0,04 & 1,00 & 0,31 & 0,19 & 1,40 & 1,91 \\
\hline Triumfetta rhomboidea Jacq. & Tiliaceae & 1 & 1 & 0,01 & 0,04 & 1,00 & 0,31 & 0,19 & 1,40 & 1,91 \\
\hline Youngia japonica (L.) DC. & Asteraceae & 1 & 1 & 0,01 & 0,04 & 1,00 & 0,31 & 0,19 & 1,40 & 1,91 \\
\hline $\begin{array}{rr}\text { Total } \\
\end{array}$ & & & & 3,19 & 21,20 & 71,18 & 100,00 & 100,00 & 100,00 & 300,00 \\
\hline
\end{tabular}

№ de Par. = número de parcelas; № de Indiv. = número de indivíduos; Fre = freqüência; Den $=$ densidade; Abu $=$ abundância; Frr $=$ freqüência relativa; Der = densidade relativa; $\mathrm{Abr}=$ abundância relativa; IVI = índice de valor de importância. 
Tabela 6 - Espécies de plantas daninhas encontradas em pastagens formadas nas várzeas do Rio São Francisco, município de Pompéu, Minas Gerais

\begin{tabular}{|c|c|c|c|c|c|c|c|c|c|c|}
\hline Espécie & Família & $\begin{array}{l}N^{o} \text { de } \\
\text { Parc. }\end{array}$ & $\begin{array}{l}\mathrm{N}^{\mathrm{o}} \text { de } \\
\text { Indiv. }\end{array}$ & Fre & Den & Abu & Frr & Der & Abr & IVI \\
\hline Echinochloa crus-galli (L.) P. Beauv. & Poaceae & 21 & 70 & 0,21 & 0,70 & 3,33 & 12,57 & 24,05 & 5,64 & 42,27 \\
\hline Vernonia polyanthes Less.. & Asteraceae & 11 & 32 & 0,11 & 0,32 & 2,91 & 6,59 & 11,00 & 4,92 & 22,51 \\
\hline Sida linifolia Juss. ex Cav. & Malvaceae & 1 & 9 & 0,01 & 0,09 & 9,00 & 0,60 & 3,09 & 15,23 & 18,92 \\
\hline Ageratum conyzoides $\mathrm{L}$. & Asteraceae & 7 & 23 & 0,07 & 0,23 & 3,29 & 4,19 & 7,90 & 5,56 & 17,66 \\
\hline Chamaesyce hirta (L.) Mill. & Euphorbiaceae & 10 & 12 & 0,10 & 0,12 & 1,20 & 5,99 & 4,12 & 2,03 & 12,14 \\
\hline Senna obtusifolia (L.) H.S. Irwin \& Barneby & Leguminosae-Caes. & 7 & 13 & 0,07 & 0,13 & 1,86 & 4,19 & 4,47 & 3,14 & 11,80 \\
\hline Sida urens L. & Malvaceae & 10 & 11 & 0,10 & 0,11 & 1,10 & 5,99 & 3,78 & 1,86 & 11,63 \\
\hline Ipomoea purpurea (L.) Roth & Convolvulaceae & 6 & 11 & 0,06 & 0,11 & 1,83 & 3,59 & 3,78 & 3,10 & 10,48 \\
\hline Emilia sonchifolia $($ L.) DC. & Asteraceae & 8 & 8 & 0,08 & 0,08 & 1,00 & 4,79 & 2,75 & 1,69 & 9,23 \\
\hline Bidens pilosa $\mathrm{L}$. & Asteraceae & 6 & 9 & 0,06 & 0,09 & 1,50 & 3,59 & 3,09 & 2,54 & 9,22 \\
\hline Mimosa pigra $\mathrm{L}$. & Leguminosae-Mim. & 7 & 8 & 0,07 & 0,08 & 1,14 & 4,19 & 2,75 & 1,93 & 8,87 \\
\hline Solanum palinacanthum Dunal & Solanaceae & 7 & 8 & 0,07 & 0,08 & 1,14 & 4,19 & 2,75 & 1,93 & 8,87 \\
\hline Porophylum ruderale (Jacq.) Cass. & Asteraceae & 6 & 7 & 0,06 & 0,07 & 1,17 & 3,59 & 2,41 & 1,97 & 7,97 \\
\hline Stachytarpheta cayennensis (Rich.)M. Vahl & Verbenaceae & 6 & 6 & 0,06 & 0,06 & 1,00 & 3,59 & 2,06 & 1,69 & 7,35 \\
\hline Solanum aculeatissimum Jacq. & Solanaceae & 5 & 6 & 0,05 & 0,06 & 1,20 & 2,99 & 2,06 & 2,03 & 7,09 \\
\hline Eclipta alba (L.) Hassk. & Asteraceae & 5 & 5 & 0,05 & 0,05 & 1,00 & 2,99 & 1,72 & 1,69 & 6,40 \\
\hline Hyparrhenia rufa (Ness) Stapf & Poaceae & 4 & 5 & 0,04 & 0,05 & 1,25 & 2,40 & 1,72 & 2,12 & 6,23 \\
\hline Triumfetta semitriloba Jacq. & Tiliaceae & 2 & 4 & 0,02 & 0,04 & 2,00 & 1,20 & 1,37 & 3,38 & 5,96 \\
\hline Blainvillea biaristata $\mathrm{DC}$. & Asteraceae & 4 & 4 & 0,04 & 0,04 & 1,00 & 2,40 & 1,37 & 1,69 & 5,46 \\
\hline Leonotis nepetaefolia (R. Br.) W.T. Aiton & Lamiaceae & 4 & 4 & 0,04 & 0,04 & 1,00 & 2,40 & 1,37 & 1,69 & 5,46 \\
\hline Desmodium barbatum (L.) Benth. & Leguminosae-Pap. & 3 & 4 & 0,03 & 0,04 & 1,33 & 1,80 & 1,37 & 2,26 & 5,43 \\
\hline Hyptis suaveolens (L.) Poit. & Lamiaceae & 3 & 4 & 0,03 & 0,04 & 1,33 & 1,80 & 1,37 & 2,26 & 5,43 \\
\hline Sorghum arundinaceum (Desv.) Stapf & Poaceae & 2 & 3 & 0,02 & 0,03 & 1,50 & 1,20 & 1,03 & 2,54 & 4,77 \\
\hline Buddleja brasiliensis Jacq.ex Spreng. & Buddlejaceae & 1 & 2 & 0,01 & 0,02 & 2,00 & 0,60 & 0,69 & 3,38 & 4,67 \\
\hline Desmodium incanum DC. & Leguminosae-Pap. & 1 & 2 & 0,01 & 0,02 & 2,00 & 0,60 & 0,69 & 3,38 & 4,67 \\
\hline Solanum americanum Mill. & Solanaceae & 1 & 2 & 0,01 & 0,02 & 2,00 & 0,60 & 0,69 & 3,38 & 4,67 \\
\hline Cleome spinosa Jacq. & Capparaceae & 3 & 3 & 0,03 & 0,03 & 1,00 & 1,80 & 1,03 & 1,69 & 4,52 \\
\hline Eupatorium pauciflorum Kunth & Asteraceae & 3 & 3 & 0,03 & 0,03 & 1,00 & 1,80 & 1,03 & 1,69 & 4,52 \\
\hline Richardia brasiliensis Gómes & Rubiaceae & 3 & 3 & 0,03 & 0,03 & 1,00 & 1,80 & 1,03 & 1,69 & 4,52 \\
\hline Waltheria americana $\mathrm{L}$. & Sterculiaceae & 3 & 3 & 0,03 & 0,03 & 1,00 & 1,80 & 1,03 & 1,69 & 4,52 \\
\hline Elvira biflora (L.) DC. & Asteraceae & 2 & 2 & 0,02 & 0,02 & 1,00 & 1,20 & 0,69 & 1,69 & 3,58 \\
\hline Cucumis anguria $\mathrm{L}$. & Cucurbitaceae & 1 & 1 & 0,01 & 0,01 & 1,00 & 0,60 & 0,34 & 1,69 & 2,63 \\
\hline Digitaria sanguinalis (L.) Scop. & Poaceae & 1 & 1 & 0,01 & 0,01 & 1,00 & 0,60 & 0,34 & 1,69 & 2,63 \\
\hline Ipomoea cairica (L.) Sweet & Convolvulaceae & 1 & 1 & 0,01 & 0,01 & 1,00 & 0,60 & 0,34 & 1,69 & 2,63 \\
\hline Momordica charantia L. & Cucurbitaceae & 1 & 1 & 0,01 & 0,01 & 1,00 & 0,60 & 0,34 & 1,69 & 2,63 \\
\hline Sida carpinifolia L. f. & Malvaceae & 1 & 1 & 0,01 & 0,01 & 1,00 & 0,60 & 0,34 & 1,69 & 2,63 \\
\hline Total & & & & 1,67 & 2,91 & 59,09 & 100,00 & 100,00 & 100,00 & 300,00 \\
\hline
\end{tabular}

№ de Par. = número de parcelas; № de Indiv. = número de indivíduos; Fre = freqüência; Den $=$ densidade; Abu $=$ abundância; Frr $=$ frequiência relativa; Der = densidade relativa; $\mathrm{Abr}=$ abundância relativa; IVI = índice de valor de importância. 
Tabela 7 - Espécies de plantas daninhas encontradas em pastagens formadas nas várzeas do Rio São Francisco, município de Abaeté, Minas Gerais

\begin{tabular}{|c|c|c|c|c|c|c|c|c|c|c|}
\hline Espécie & Família & $\begin{array}{l}\mathrm{N}^{\mathrm{o}} \text { de } \\
\text { Parc. }\end{array}$ & $\begin{array}{l}\mathrm{N}^{\mathrm{o}} \mathrm{de} \\
\text { Indiv. }\end{array}$ & Fre & Den & Abu & Frr & Der & Abr & IVI \\
\hline Hypenia densiflora (Pohl ex Benth.) Harl. & Lamiaceae & 68 & 85 & 0,68 & 3,40 & 1,25 & 16,71 & 14,96 & 1,79 & 33,46 \\
\hline Sida urens L. & Malvaceae & 55 & 60 & 0,55 & 2,40 & 1,09 & $13,51 \ldots$ & 10,56 & 1,56 & 25,64 \\
\hline Conyza bonariensis (L.) Cronquist & Asteraceae & 27 & 82 & 0,27 & 3,28 & 3,04 & 6,63 & 14,44 & 4,35 & 25,42 \\
\hline Ipomoea grandifolia (Dammel) O'Donel & Convolvulaceae & 43 & 48 & 0,43 & 1,92 & 1,12 & 10,57 & 8,45 & 1,60 & 20,62 \\
\hline Ageratum conyzoides L. & Asteracee & 5 & 26 & 0,05 & 1,04 & 5,20 & 1,23 & 4,58 & 7,45 & 13,26 \\
\hline Corchorus hirtus $\mathrm{L}$. & Tiliaceae & 17 & 32 & 0,17 & 1,28 & 1,88 & 4,18 & 5,63 & 2,70 & 12,51 \\
\hline $\begin{array}{l}\text { Alternanthera tenella Colla } \\
\text { Ala }\end{array}$ & Amaranthaceae & 21 & 22 & 0,21 & 0,88 & 1,05 & 5,16 & 3,87 & 1,50 & 10,53 \\
\hline Desmodium incanum DC. & Leguminosae-Pap. & 1 & 6 & 0,01 & 0,24 & 6,00 & 0,25 & 1,06 & 8,60 & 9,90 \\
\hline Sida rhombifolia L. & Malvaceae & 7 & 16 & 0,07 & 0,64 & 2,29 & 1,72 & 2,82 & 3,28 & 7,81 \\
\hline Dichondra repens J.R.Forst. \& G. Forst. & Convolvulaceae & 14 & 15 & 0,14 & 0,60 & 1,07 & 3,44 & 2,64 & 1,54 & 7,62 \\
\hline Heliotropium indicum L. & Boraginaceae & 12 & 15 & 0,12 & 0,60 & 1,25 & 2,95 & 2,64 & 1,79 & 7,38 \\
\hline Acanthospermum hispidum DC. & Asteraceae & 11 & 11 & 0,11 & 0,44 & 1,00 & 2,70 & 1,94 & 1,43 & 6,07 \\
\hline Stachytarpheta cayennensis (Rich.) M.Vahl. & Verbenaceae & 9 & 11 & 0,09 & 0,44 & 1,22 & 2,21 & 1,94 & 1,75 & $\begin{array}{l}5,90 \\
5,90 \ldots\end{array}$ \\
\hline Hyptis spicigera Lam. & Lamiaceae & 7 & 10 & 0,07 & 0,40 & 1,43 & 1,72 & 1,76 & 2,05 & 5,53 \\
\hline 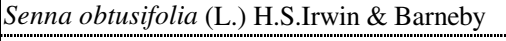 & Leguminosae-Caes. & 8 & 10 & 0,08 & 0,40 & 1,25 & 1,97 & 1,76 & 1,79 & $\begin{array}{l}5,52 \\
5,52\end{array}$ \\
\hline Sida viarum A. St. Hil. & Malvaceae & 3 & 7 & 0,03 & 0,28 & 2,33 & 0,74 & 1,23 & 3,34 & 5,31 \\
\hline Eclipta alba (L.) Hassk. & Asteraceae & 9 & 9 & 0,09 & 0,36 & 1,00 & 2,21 & 1,58 & 1,43 & 5,23 \\
\hline Sida cordifolia $\mathrm{L}$ & Malvaceae & 1 & 3 & 0,01 & 0,12 & 3,00 & 0,25 & 0,53 & 4,30 & 5,07 \\
\hline Bidens pilosa $\mathrm{L}$. & Asteraceae & 6 & 8 & 0,06 & 0,32 & 1,33 & 1,47 & 1,41 & 1,91 & 4,79 \\
\hline Blainvillea rhomboidea Cass. & Asteraceae & 5 & 7 & 0,05 & 0,28 & 1,40 & 1,23 & 1,23 & 2,01 & 4,47 \\
\hline Commelina benghalensis L. & Commelinaceae & 7 & 7 & 0,07 & 0,28 & 1,00 & 1,72 & 1,23 & 1,43 & 4,39 \\
\hline $\begin{array}{l}\text { Vernonia polyanthes Lees. } \\
\text { Vernon }\end{array}$ & Asteraceae & 3 & 5 & 0,03 & 0,20 & 1,67 & 0,74 & 0,88 & 2,39 & $\begin{array}{l}4,01 \\
4,01\end{array}$ \\
\hline $\begin{array}{l}\text { Eleusine indica (L.) Gaertn. } \\
\text { Elene indica }\end{array}$ & Poaceae & 5 & 6 & 0,05 & 0,24 & 1,20 & 1,23 & 1,06 & 1,72 & 4,00 \\
\hline $\begin{array}{l}\text { Phyllanthus tenellus Roxb. } \\
\text { Phys }\end{array}$ & Euphorbiaceae & 5 & 6 & 0,05 & 0,24 & 1,20 & 1,23 & 1,06 & 1,72 & 4,00 \\
\hline Hyptis suaveolens (L.) Poit. & Lamiaceae & 6 & 6 & 0,06 & 0,24 & 1,00 & 1,47 & 1,06 & 1,43 & 3,96 \\
\hline 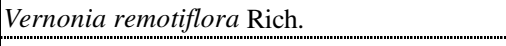 & Asteraceae & 6 & 6 & 0,06 & 0,24 & 1,00 & 1,47 & 1,06 & 1,43 & 3,96 \\
\hline Leonotis nepetaefolia (R.Br.) W.T.Aiton & Lamiaceae & 5 & 5 & 0,05 & 0,20 & 1,00 & 1,23 & 0,88 & 1,43 & 3,54 \\
\hline Asclepias curassavica $\mathrm{L}$. & Asclepiadaceae & 2 & 3 & 0,02 & 0,12 & 1,50 & 0,49 & 0,53 & 2,15 & 3,17 \\
\hline Solanum sisymbriifolium Lam. & Solanaceae & 2 & 3 & 0,02 & 0,12 & 1,50 & 0,49 & 0,53 & 2,15 & 3,17 \\
\hline Wissadula subpeltata (Kuntze.) R.E.Fr. & Malvaceae & 2 & 3 & 0,02 & 0,12 & 1,50 & 0,49 & 0,53 & 2,15 & 3,17 \\
\hline 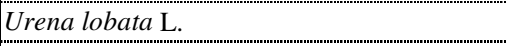 & Malvaceae & 4 & 4 & 0,04 & 0,16 & 1,00 & 0,98 & 0,70 & 1,43 & 3,12 \\
\hline $\begin{array}{l}\text { Emilia sonchifolia }(\mathrm{L} .) \text { DC. } \\
\text { Emila }\end{array}$ & Asteraceae & 3 & 3 & 0,03 & 0,12 & 1,00 & 0,74 & 0,53 & 1,43 & 2,70 \\
\hline Scoparia dulcis $\mathrm{L}$ & Scrophulariaceae & 3 & 3 & 0,03 & 0,12 & 1,00 & 0,74 & 0,53 & 1,43 & 2,70 \\
\hline Sida spinosa $\mathrm{L}$. & Malvaceae & 3 & 3 & 0,03 & 0,12 & 1,00 & 0,74 & 0,53 & 1,43 & 2,70 \\
\hline Sidastrum micranthum (A.St.Hil.) Fryxel & Malvaceae & 3 & 3 & 0,03 & 0,12 & 1,00 & 0,74 & 0,53 & 1,43 & 2,70 \\
\hline Solanum americanum Mill. & Solanaceae & 3 & 3 & 0,03 & 0,12 & 1,00 & 0,74 & 0,53 & 1,43 & 2,70 \\
\hline $\begin{array}{l}\text { Aeschynomene falcata (Poir) DC. } \\
\text { Aeschonen }\end{array}$ & Leguminosae-Pap. & 2 & 2 & 0,02 & 0,08 & 1,00 & 0,49 & 0,35 & 1,43 & 2,28 \\
\hline 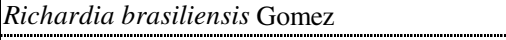 & Rubiaceae & 2 & 2 & 0,02 & 0,08 & 1,00 & 0,49 & 0,35 & 1,43 & 2,28 \\
\hline Triumfetta semitriloba Jacq. & Tiliaceae & 2 & 2 & 0,02 & 0,08 & 1,00 & 0,49 & 0,35 & 1,43 & 2,28 \\
\hline Waltheria americana $\mathrm{L}$. & Sterculiaceae & 2 & 2 & 0,02 & 0,08 & 1,00 & 0,49 & 0,35 & 1,43 & 2,28 \\
\hline Amaranthus spinosus L. & Amaranthaceae & 1 & 1 & 0,01 & 0,04 & 1,00 & 0,25 & 0,18 & 1,43 & 1,86 \\
\hline Crotalaria lanceolata E. Mey & Leguminosae-Pap. & 1 & 1 & 0,01 & 0,04 & 1,00 & 0,25 & 0,18 & 1,43 & 1,86 \\
\hline Cuphea cartagenensis (Jacq.) J.F. Macbr. & Lythraceae & 1 & 1 & 0,01 & 0,04 & 1,00 & 0,25 & 0,18 & 1,43 & 1,86 \\
\hline 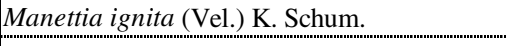 & Rubiaceae & 1 & 1 & 0,01 & 0,04 & 1,00 & 0,25 & 0,18 & 1,43 & 1,86 \\
\hline $\begin{array}{l}\text { Porophylum ruderale (Jacq.) Cass. } \\
0\end{array}$ & Asteraceae & 1 & 1 & 0,01 & 0,04 & 1,00 & 0,25 & 0,18 & 1,43 & 1,86 \\
\hline Solanum aculeatissimum Jacq. & Solanaceae & 1 & 1 & 0,01 & 0,04 & 1,00 & 0,25 & 0,18 & 1,43 & 1,86 \\
\hline Stylosanthes guianensis (Aubl.) Sw. & Leguminosae-Pap. & 1 & 1 & 0,01 & 0,04 & 1,00 & 0,25 & 0,18 & 1,43 & 1,86 \\
\hline Zornia latifolia $\mathrm{Sm}$. & Leguminosae-Pap. & 1 & 1 & 0,01 & 0,04 & 1,00 & 0,25 & 0,18 & 1,43 & 1,86 \\
\hline Total & & & & 4,07 & 22,72 & 69,77 & 100,00 & 100,00 & 100,00 & 300,00 \\
\hline
\end{tabular}

№ de Par. = número de parcelas; № de Indiv. = número de indivíduos; Fre $=$ freqüência; Den $=$ densidade; Abu $=$ abundância; Frr $=$ freqüência relativa; Der $=$ densidade relativa; $\mathrm{Abr}=$ abundância relativa; IVI = índice de valor de importância. 
Tabela 8 - Índice de Similaridade de SФrensen da composição de plantas daninhas em pastagens de várzeas, entre as localidades estudadas (\%)

\begin{tabular}{|l|c|c|c|c|c|}
\hline \multicolumn{1}{|c|}{ Localidade } & Iguatama & $\begin{array}{c}\text { Bom } \\
\text { Despacho }\end{array}$ & $\begin{array}{c}\text { Martinho } \\
\text { Campos }\end{array}$ & Luz & Pompéu \\
\hline Iguatama & 16,47 & & & & \\
Bom Despacho & 22,88 & 25,44 & & & \\
Martinho Campos & 29,88 & 20,67 & 29,52 & 25,67 & \\
Luz & 22,67 & 19,13 & 25,13 & 28,83 & 21,00 \\
Pompéu & 33,13 & 37,00 & 20,20 & & \\
Abaeté &
\end{tabular}

Ocorreram poucas espécies dominantes, o que permite utilizar, em cada local, herbicidas específicos para controlar apenas as espécies de maior ocorrência, reduzindo assim o uso de herbicidas e mantendo uma comunidade de plantas invasoras em niveis que não afetam a produção e que possam sustentar uma variabilidade de insetos benéficos ao agroecossistema (Macedo \& Martins, 1998, 1999).

\section{LITERATURA CITADA}

BRANDÃO, M.; BRANDÃO, H.; LACA-BUENDIA, J. P. A mata ciliar do Rio Sapucaí, município de Santa Rita do Sapucaí-MG: fitossociologia. Daphne, v. 8, n. 4, p. 36-48, 1998.

MACEDO, J. F.; MARTINS, R. P. Potencial da erva daninha Waltheria americana L. (Sterculiaceae) no manejo integrado de pragas e polinizadores: visitas de abelhas e vespas. An. Soc. Entol. Brasil, v. 27, n. 1, p. 29-40, 1998.
MACEDO, J. F.; MARTINS, R. P. A estrutura da guilda de abelhas e vespas visitantes florais de Waltheria americana $\mathrm{L}$. (Sterculiaceae). An. Soc. Entol. Brasil, v. 28, n. 4, p. 617633, 1999.

MASCARENHAS, M. H. T. et al. Plantas daninhas raramente mencionadas como infestantes de várzeas em Minas Gerais - Brasil. Daphne, v. 5, n. 1, p. 72-78, 1995.

MASCARENHAS, M. H. T. et al. Poáceas, Fabáceas, Asteráceas e Cyperaceas infestantes de várzeas em Minas Gerais - Brasil. Daphne, v. 2, n. 4, p. 37-43, 1992.

CUNHA, L. H.; BRANDÃO, M. Plantas daninhas em culturas em áreas de várzeas no município de Governador Valadares, MG. Daphne, v. 7, n. 1, p. 60-68, 1997.

SФRENSEN, T. A method of stablishing groups of equal amplitude in plant society based on similarity of species content. In: ODUN, E. P. (Ed.). Ecologia. 3.ed. México: Interamericana, 1972. $640 \mathrm{p}$. 